GRANDE-BRETAGNE

\section{LA POLLUTION PLUVIALE EN QUESTION}

\author{
Edith FLORET-MIGUET * et \\ Bernard BARRAQUE ** \\ * Doctorante, LATTS, ENPC. \\ ** Chercheur CNRS au LATTS.
}

\section{INTRODUCTION}

Le réseau public britannique comprend $300630 \mathrm{~km}$ de canalisations [1] ; il est composé à $70 \%$ de réseaux unitaires [2] et comptait 17719 déversoirs d'orage en réseaux unitaire et en stations d'épuration en 1991 [3].

En 1989, une enquête [4] répertoriait plus de 29000 points de déversement, y compris 8000 déversoirs de réseaux pluviaux. Ces points de déversement étaient, selon B. Ellis [5], les principaux responsables de la dégradation de la qualité des rivières, qui, en 1985 , comptaient, pour la première fois depuis 1958 [6], plus de $10 \%$ de cours d'eau classés comme étant pollués à fortement pollués.

B. Ellis considérait la pollution causée par les déversoirs d'orage des réseaux unitaires comme étant la plus dommageable mais il reconnaissait que la source de pollution la plus commune due aux égouts publics provenait des déversements des réseaux pluviaux. En 1992, cela semble avoir été oublié par certains.

"Pourquoi traiter les eaux pluviales? Elles sont propres!"

$\mathrm{Ce}$ cri du cœur d'un ingénieur d'une des compagnies des eaux britanniques illustre assez bien la conception des eaux pluviales encore couramment répandue au Royaume-Uni. La plupart du temps, les eaux strictement pluviales ne sont considérées comme un problème qu'en tant que risque d'inondation. Dans le pays où s'est manifestée en premier l'attitude civilisée la plus scrupuleuse à l'égard de l'hygiène, le mot "pollution " reste associé aux eaux usées. De même, dans la Directive Européenne sur les Eaux Usées du 21 mai 1991, la pollution pluviale n'est pas reconnue en elle-même mais uniquement au travers des rejets des déversoirs d'orage des réseaux unitaires. La Directive est centrée sur les stations d'épuration classiques, alors même qu'elle était largement motivée par l'eutrophisation des grands fleuves et de la Mer du Nord, due pour une bonne part au ruissellement pluvial urbain et rural. Cette part déterminante de la pollution a été officiellement reconnue aux USA par l'EPA cette année. Mais, pour que des actions spécifiques soient entreprises, il faudrait réorienter l'action des ingénieurs, des deux côtés de l'Atlantique...

\section{UN MANQUE DE CONSIDÉRATION POUR LA POLLUTION DES EAUX STRICTEMENT PLUVIALES}

En Grande-Bretagne, le manque de considération pour la pollution des eaux strictement pluviales se traduit par une absence quasi-générale de dispositifs de traitement des eaux transitant par les réseaux pluviaux.

Ponctuellement, on peut trouver des sites où les eaux pluviales font l'objet de stockages ou d'une épuration. Mr. Trabuc [7] cite le cas de certaines grandes agglomérations de la Severn Trent region et de deux villes nouvelles (Milton Keynes et Stevenage) qui utilisent les eaux de ruissellement urbain pour créer des plans d'eau à usage récréatif. Des dispositifs conçus pour piéger les hydrocarbures sont obligatoires pour certaines activités (garage, industrie...), des chambres de sédimentation et d'élimination d'hydrocarbures existent également pour recueillir les eaux ruisselant sur les chaussées. Ces systèmes permettent effectivement de traiter les eaux de ruissellement s'ils sont bien entretenus, ce qui ne semble pas toujours être le cas, notamment quand les autorités locales connaissent des difficultés financières [8]. Des techniques de stockage en réseau sont également utilisées pour diminuer les risques d'inondation, mais sans effet réel sur la qualité des effluents rejetés.

L'utilisation de bassins de retenue urbains pour le contrôle des inondations et l'atténuation des charges polluantes a certes été prise en compte mais n'a pas toujours été bien acceptée par les Autorités Régionales de l'Eau. Ainsi, North West Water déconseillait officieusement l'adoption de "petits " bassins de retenue en dépit de leurs avantages tant pour la prévention des inondations que pour la protection du milieu (Ellis, 1989). En effet, un bassin de retenue revient cher en construction et en entretien. Or, il existait (et c'est encore le cas) de grandes incertitudes sur la détermination et la répartition des responsabilités financières, opérationnelles et d'entretien des bassins entre le promoteur, l'autorité locale et l'exploitant des réseaux.

Les méthodes d'infiltration in situ, qui constituent, lorsque les conditions géologiques le permettent, les moyens les plus efficaces actuellement pour contrôler les débits et la qualité des écoulements pluviaux, sont rarement utilisées. En 1988, beaucoup d'ingénieurs en exercice dans les Autorités Régionales de l'Eau et les autorités locales étaient très sceptiques à leur sujet (Ellis, 1989). Et en 1990, Ellis [9] citait le cas de gestionnaires d'autoroute qui refusaient d'adopter des puisards ou des procédés de biofiltration pour traiter les eaux de ruissellement et imposaient un déversoir.

Le manque de prise en considération de la pollution des eaux strictement pluviales se traduit également par l'absence de réglementation en ce qui concerne les réseaux pluviaux.

Ainsi les responsabilités de la National Rivers Authority (NRA) couvrent toutes les eaux: rivières, lacs, réservoirs, estuaires, eaux côtières ( 3 miles), et eaux souterraines. D'après les termes de la Loi sur l'Eau, ce sont des " eaux contrôlées " et occasionner "l'introduction de toute substance toxique, nocive ou polluante ou de tout déchet solide dans les eaux contrôlées " est un délit. II est donc interdit d'effectuer un déversement sans l'autorisation préalable de la NRA. Cette disposition concerne a priori les rejets des stations d'épuration, des déversoirs d'orage des réseaux unitaires, des déversoirs d'urgence des stations, et les rejets en mer, mais aussi les déversements des réseaux pluviaux.

Or, en compulsant The Water Share Offers, publié pour préparer la mise en vente des actions des Autorités Régionales de l'Eau en 1989, nous nous sommes aperçus que les déversements des collecteurs pluviaux ne sont que rarement soumis à l'obligation de répondre aux normes fixées par une autorisation de la NRA : six des dix autorités de l'eau n'avaient même pas répertorié les points de déversements des eaux strictement pluviales, et parmi ceux répertoriés, seulement $11 \%$ faisaient l'objet d'un contrôle [10]!

Le cri du cœur de l'ingénieur britannique ci-dessus cité révèle ainsi que les compagnies des eaux ne sont pas encore prêtes à allouer des fonds au traitement des eaux des réseaux pluviaux. Tant que des autorisations de rejets ne seront pas instaurées, elles ne seront pas contraintes à respecter une norme de rejet, aussi l'installation d'ouvrages d'épuration dans les réseaux séparatifs ne leur semblera pas nécessaire. Les moyens de financement seront réservés aux travaux sur les stations d'épuration et les déversoirs d'orage qui, eux, font l'objet d'autorisations de rejet et peuvent, en cas de nonconformité avec les normes, provoquer la mise à l'amende de l'exploitant. 


\section{LES TECHNIQUES UTILISÉES POUR TRAITER LES EAUX DE PLUIE}

\subsection{EN RÉSEAU UNITAIRE}

La pollution due aux déversoirs d'orage a, elle, été depuis longtemps identifiée comme un problème dans l'histoire des systèmes d'assainissement britanniques. Un rapport de la Commission Royale publié en 1908 recommandait déjà une utilisation modérée des déversoirs d'orage. Et en 1970, le rapport final du Comité Technique sur les Déversoirs d'Orage reconnaissait " le besoin urgent d'une étude sur les effets des rejets intermittents des déversoirs dans les cours d'eau ".

Dans l'optique d'une protection du milieu naturel, ces rapports ont établi des normes de dimensionnement des déversoirs d'orage. Ceux-ci sont construits pour éviter tout déversement à la rivière tant que le flux dans l'égout ne représente pas au moins six fois le débit maximum par temps sec (Royal Commission 1908), voire neuf fois pour les déversoirs construits ou réhabilités récemment suivant les recommandations du rapport final du Technical Committee on Storm Overflows and Disposal of Storm Sewage (1970).

Des mesures ont été prises dès les années 70 pour tenter de résoudre le problème posé par les rejets pluviaux des réseaux unitaires et elles ont été détaillées dans le Sewerage Rehabilitation Manual (SRM ; 1983 - révisé en 1986). Les premières voies choisies pour maîtriser les surverses unitaires se sont appuyées sur la réduction des débits de pointe en amont des réseaux (bassins de rétention), ce qui présentait l'avantage de gagner sur le coût des ouvrages aval et d'apporter accessoirement un abattement de pollution. Une solution d'abord complémentaire, puis plus largement développée, a consisté à installer des stockages en différents points du réseau afin de réduire la fréquence de fonctionnement des déversoirs d'orage.

Cherchant à jouer sur les capacités de stockage en réseau, les ingénieurs ont ainsi préféré les déversoirs latéraux à seuil haut, les chambres de décantation et les déversoirs de type Vortex (séparateur tourbillonnaire) [11] aux déversoirs utilisés antérieurement (déversoir latéral à seuil bas, déversoir à saut...) qui présentaient de faibles caractéristiques hydrauliques et étaient peu performants [12]. Ces équipements permettent en outre une légère atténuation de la pollution véhiculée par les eaux pluviales.

Actuellement, pour éviter le rejet d'éléments flottants et, à la suite de recherches récentes, la NRA veut transformer les normes de dimensionnement des déversoirs d'orage pour éviter toute surverse tant que le débit n'atteint pas douze fois celui par temps sec. Mais cette proposition soulève des protestations dans les compagnies des eaux qui veulent prouver que le dimensionnement actuel des déversoirs est adapté à la protection du milieu.

Pourtant, aujourd'hui encore, entre 25 et $35 \%$ des déversoirs d'orage débouchant sur un cours d'eau sont supposés être préjudiciables au milieu naturel (ELLIS, 1991). II faut dire que le SRM cherche à résoudre les deux problèmes liés aux eaux pluviales : la protection contre les inondations et la préservation du milieu. Or, cette protection du milieu reste relative car ces solutions techniques entrent en action alors que les eaux sont déjà polluées ; et elles n'offrent alors, en général, qu'une faible capacité de stockage comparée au volume qu'il faudrait traiter pour éviter toute pollution du milieu récepteur. En outre, la priorité reste la lutte contre les inondations et, pour cela, la solution la plus simple est l'écoulement rapide des effluents vers le milieu naturel...

\subsection{EN STATION}

Les Britanniques se sont néanmoins équipés de moyens pour traiter directement les eaux pluviales et ce, en un lieu qui soulève de grandes controverses en France, puisqu'il s'agit des stations d'épuration des eaux résiduaires urbaines.
Cette pratique doit, cependant, pour être mieux comprise, être mise en parallèle avec la composition du parc de stations britannique. Les Lits Bactériens, filière de traitement des moins sensibles aux variations de charges - première conséquence d'un événement pluvieux - prédominent, semble-t-il, sur toute autre technique de traitement. Nous avons en effet pu reconstituer l'état d'une partie du parc (2 820 stations sur 6 400) [13] : et l'homogénéité de la répartition en filières de traitement (cf. figure 1) nous permet d'avancer que les Lits Bactériens occupent près de $70 \%$ du parc britannique.

Tableau 1: Répartition des stations d'épuration par filière de traitement (1992)

\begin{tabular}{|c|c|c|}
\hline Filière & Nombre & $\% / 2820$ \\
\hline Lits Bactériens & 2081 & $73,79 \%$ \\
\hline Boues Activées & 247 & $8,76 \%$ \\
\hline Autres & 492 & $17,45 \%$ \\
\hline
\end{tabular}

Lorsque la station est dimensionnée pour plus de 10000 eq. hab., des bassins d'orage permettent la décantation de la part des écoulements compris entre trois fois et jusqu'à neuf fois le débit par temps sec, les eaux décantées étant généralement traitées à la fin de l'événement pluvieux. Ces stations sont conçues pour traiter complètement un volume égal à trois fois le débit par temps sec. Le nombre de bassins est généralement supérieur ou égal à deux, et ils se remplissent en série afin d'allonger le temps de rétention. Lorsque l'orage dure et que le cours d'eau récepteur ne présente pas un débit suffisant pour la dilution des effluents, les éventuels déversements sont dirigés vers des marais afin de leur apporter un traitement complémentaire.

Dans les stations de moins de 10000 eq. hab., il existe peu de bassins d'orage. L'usage, par temps de pluie, est de traiter les eaux jusqu'à ce que le débit atteigne 3 fois le débit par temps sec. L'absence de bassin d'orage facilite en effet la construction et l'exploitation de la station, et, surtout, " l'équipe mobile (de maintenance) est réduite " [14].

Ainsi, les Britanniques appliquent déjà en partie les rares recommandations de la Directive Européenne, du 21 mai 1991 relative aux Eaux Résiduaires Urbaines, concernant les eaux pluviales (cf. Annexe 1); à savoir le traitement en station du mélange eaux usées - eaux pluviales sauf en cas de "précipitations exceptionnellement fortes " .

II semblerait même que certains songent à aller encore plus loin dans ce domaine. II existe, en effet, un projet de recherche au sein du Water Research center (WRc, le laboratoire de recherches le plus important dans le domaine de l'assainissement) sur les éventuelles autorisations de rejets que pourrait imposer la NRA pour les déversoirs des réseaux pluviaux et les conséquences qu'elles auraient pour les compagnies des eaux (WSCs). Cette recherche est à peine entamée mais lon Clifforde [15] envisageait comme solution possible pour traiter les eaux pluviales, le détournement des eaux de ruissellement dans les réseaux unitaires (i.e. vers les stations d'épuration); et cela, dans le but de répondre aux attentes des WSCs. Ces dernières, en effet, veulent limiter leurs coûts d'exploitation et cherchent à concentrer leur personnel en des points précis. Elles semblent ainsi très réticentes à l'installation d'équipements sur un réseau. Même l'usage d'une simple grille au droit d'un déversoir d'orage est sujet à controverse [16] car sous-entend la nécessité d'un entretien régulier!

\section{CONCLUSION}

Ainsi, l'approche sanitaire de l'assainissement (évacuer les eaux le plus rapidement possible) a précédé et prévaut toujours sur une approche environnementale de l'eau, préoccupée par 

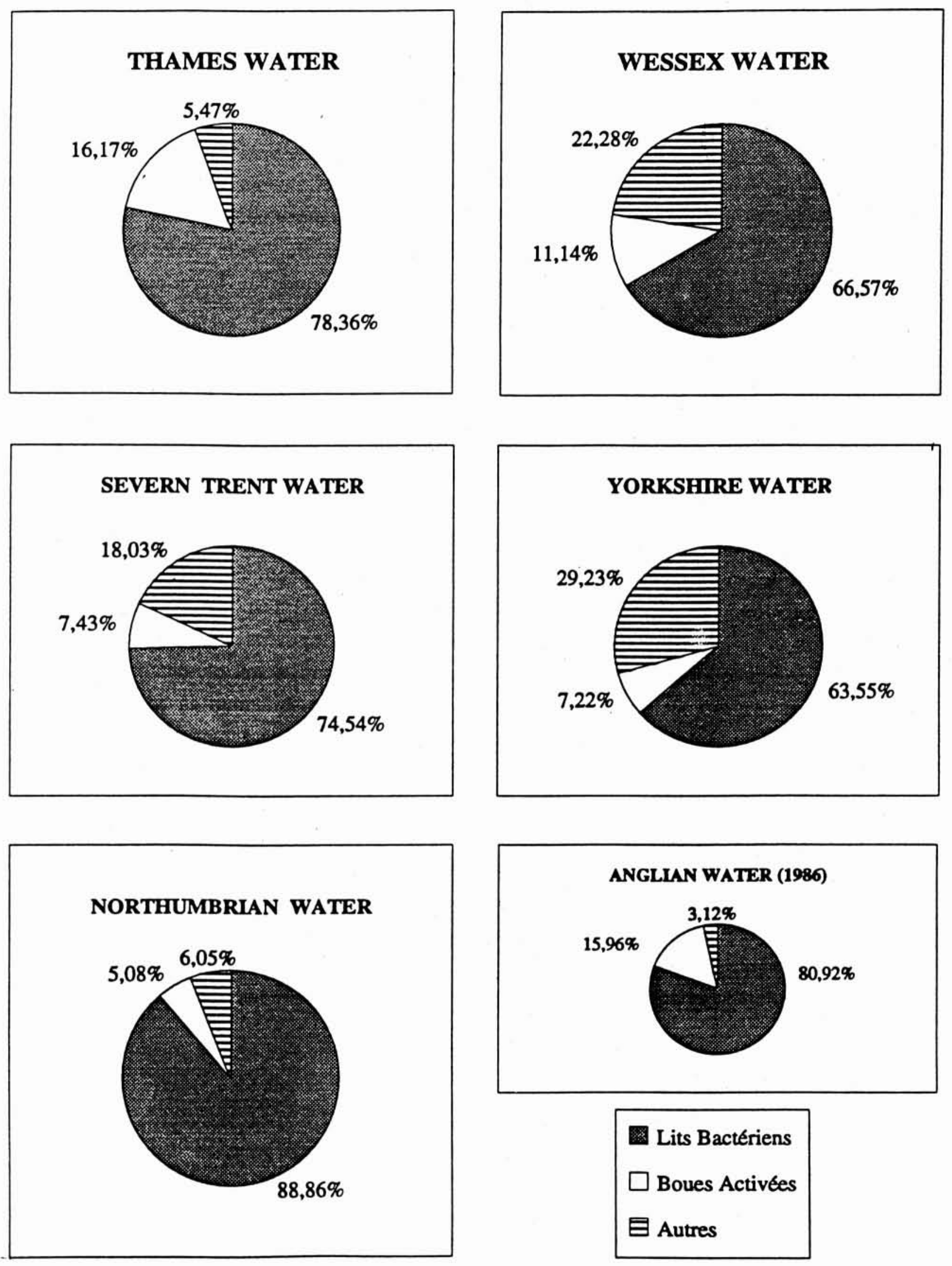

Figure 1: Répartition des parcs de stations d'èpuration par filières de traitement (1992) 
la Santé Publique mais également soucieuse de préserver le milieu naturel. On l'a dit, le mot " pollution " reste synonyme de rejet d'eaux usées et il ne faut donc pas s'étonner de constater que les réseaux pluviaux aient été peu équipés en techniques de traitement. Le système de réglementation s'est d'ailleurs focalisé sur les rejets d'eaux usées au milieu naturel.

Dans notre communication sur l'avenir des normes de qualité de l'eau aux $22^{\circ}$ Journées de l'Hydraulique [17], nous avons tenté de montrer cette difficulté de passer de l'approche sanitaire à l'approche environnementaliste de l'eau, comme en témoigne le processus d'adoption de la Directive Européenne Eaux Usées de mai 1991, mais aussi comme en témoigne la dérive du processus de normalisation de l'eau potable aux Etats-Unis. La gestion du " pluvial ", dans une approche d'hydrologie urbaine, intégrant les aspects qualitatifs et quantitatifs, est victime, au fond, du caractère traditionnel de l'approche des ingénieurs.

A bien y regarder, il y a un obstacle supplémentaire à la mise en œuvre de nouvelles politiques. Celles-ci passent, en effet, par l'imposition de contraintes nouvelles à l'urbanisation, que l'on limite l'imperméabilisation des sols ou que l'on réserve des emprises pour faire des bassins de retenue. Ces contraintes impliquent, au second degré, de réapprendre aux citadins à participer à la gestion de l'eau, là où auparavant, on les déchargeait complètement de toute tâche.

Comme il est difficile d'imposer d'emblée ces pratiques nouvelles, on cherche, dans beaucoup de pays, à s'appuyer sur des mécanismes incitatifs, notamment financiers. Mais il est bien difficile de les mettre en œuvre. On sait qu'en France, les projets de "redevances pluvial ", caressés par les Agences de l'Eau, n'ont pas été repris dans la Loi du 3 janvier 1992. En Grande-Bretagne, la situation est de ce point de vue paradoxale : en effet, le système de tarification de l'eau (système public fiscalisé) est fondé sur la taille de la propriété bâtie, et il pourrait facilement être adapté pour inclure une incitation à participer à l'effort de maîtrise des eaux de pluie. Pourtant, la loi de privatisation prévoit l'abandon de ce système au profit du comptage, comme c'est le cas général sur le continent, avec inclusion de l'assainissement dans la facture d'eau potable.

Les recherches menées par les différents laboratoires britanniques (WRc, Urban Pollution Research Center [18]) sur la pollution véhiculée par les eaux pluviales ne semblent pas avoir reçu toute l'attention nécessaire à la résolution du problème qu'elle soulève. C'est, nous le pensons, à la National Rivers Authority qu'il appartient de prendre l'initiative. Son rôle est, dans ce domaine, primordial puisque c'est elle qui, en délivrant les autorisations de rejets, peut influencer à la fois les aménageurs de sites et les compagnies des eaux. N'oublions pas, en effet, que depuis la privatisation de 1989 , le respect des Objectifs de Qualité est inscrit dans la loi et que la NRA dispose ainsi d'un pouvoir de police sur la nature des déversements au milieu naturel. La situation pourrait donc évoluer grâce à des solutions réglementaires.

Nous aimerions, à ce propos, revenir sur les voies de recherches du WRc concernant le traitement des eaux pluviales. La stratégie britannique pourrait, on l'a vu, s'orienter vers le traitement de toutes les eaux pluviales en station d'épuration (si tant est que le dimensionnement actuel des stations, même confortable, le permet).

En France, une telle option est loin de faire l'unanimité. Certes, la structure de distribution des financements entre réseau (pour ses fonctions d'assainissement) et station (pour ses fonctions de dépollution) se trouverait confortée par la construction de capacités supplémentaires de traitement en stations d'épuration. Traiter les eaux pluviales en stations respecterait la compartimentation actuelle des services assainissement et épuration et engendrerait des recettes supplémentaires pour les constructeurs de stations.

Mais, la nature de la pollution véhiculée par les eaux pluviales diffère notablement de celle des eaux usées et une station d'épuration classique n'est, de ce fait, pas forcément adaptée à ce type de traitement. Le passage en station des eaux pluviales peut même être plus dommageable que bénéfique, la qualité générale des effluents rejetés pouvant diminuer.

En Grande-Bretagne, cette option est cependant plus envisageable qu'en France, en particulier, parce que la structure du parc de stations britanniques y est un peu mieux adaptée. Le parc français de stations d'épuration est en effet dominé à près de $60 \%$ par la filière des Boues Activées [19], technique des plus sensibles aux variations de charge engendrées par un événement pluvieux...

\section{BIBLIOGRAPHIE}

[1] Waterfacts, Water Services Association, Londres, 1991.

[2] FIDDES D. - Overview of international urban drainage practice, The Public Health Engineer, 1986, 14, $n^{\circ} 3$, pp. 22-24.

[3] Waterfacts, Water Services Association, Londres, 1991.

[4] The Water Share Offers, Offers for sale by Schroders on behalf of the Secretary of State of the Environment and the Secretary of State for Wales, Department of Environment, novembre 1989.

[5] ELLIS J.B. - The management and control of urban runoff quality, Journal of the Institution of Water and Environmental Management, vol. 3, $n^{\circ} 2$, avril 1989.

[6] SHUTTLEWORTH A.J. - State of rivers and sewers in Britain - Is there a pollution problem ?. The Public Health Engineer, 1986, 14, $n^{\circ} 3$, pp. 49-51.

[7] TRABUC P. - Dépollution pluviale : politique de la Communauté Européenne et exemples nationaux, La gestion de l'eau - Colloque Européen, 4-6 décembre 1990, Paris, Presses des Ponts et Chaussées, pp. 169-183.

[8] D'après un entretien avec P. Chatfield, NRA Thames (Reading), le 30 avril 1992.

[9] ELLIS J.-B. - The control and management of urban stormwater runoff quality in the United Kingdom: a national perspective, La gestion de l'eau - Colloque Européen, 4-6 décembre 1990, Paris, Presses des Ponts et Chaussées, pp. 77-86.

[10] Contre $99 \%$ des déversoirs d'orage des réseaux unitaires et des stations d'épuration.

[11] TYSON J.M., CLIFFORDE I.T. - River impact of combined sewer overflows : UK developments, International symposium on urban hydrology and municipal engineering, 14 juin 1988, Markham, pp. F5-1-F5-11.

[12] ELLIS J.B. - Measures for the control and treatment of urban runoff quality, rapport élaboré à la demande de l'Agence de l'Eau Seine-Normandie, 1991, 135 pages.

[13] FLORET-MIGUET E. - Comparaison des politiques d'assainissement pluvial en Grande-Bretagne et en France, mémoire de DEA, ENGREF, ENPC, Paris XII, 1992.

[14] Manuals of British Practice in Water Pollution Control, Unit Processes: Preliminary Processes, IWEM, Londres, 1984, p. 48.

[15] Responsable Assainissement; propos recueillis lors d'un entretien le 29 avril 1992 à Swindon (WRc).

[16] Tyson et col., 1988, op. cit.

[17] BARRAQUE B., LAVOUX T. - Qualité de l'eau, quelles normes pour demain?, Communication aux $22^{\circ}$ Journées de l'Hydraulique, Société Hydrotechnique de France, 1992.

[18] Middlesex University, London. Voir aussi FIDDES D. et CLIFFORDE I.T. - River Basin Management: Developing the Tools, IWEM 89 Conference paper, 4 Feb. 1990, pp. 90-97.

[19] BERLAND J.-M. - Innovations technologiques en matière e stations d'épuration: analyse comparative France - République Fédérale d'Allemagne, mémoire de DEA, ENGREF, ENPC, Paris XII, 1990. 